\begin{tabular}{|c|l|}
\hline Title & Cluster-shell competition in light nuclei \\
\hline Author(s) & Itagaki, N.; A oyama, S.; Okabe, S.; Ikeda, K. \\
\hline Citation & $\begin{array}{l}\text { Physical Review C, 70, 054307 } \\
\text { https://doi.org/10.1103/PhysRevC.70.054307 }\end{array}$ \\
\hline Issue Date & 2004-11-05 \\
\hline Doc URL & http://hdl.handle.net/2115/17211 \\
\hline Rights & Copyright $\odot 2004$ American Physical Society \\
\hline Type & article \\
\hline File Information & PRC70-054307.pdf \\
\hline
\end{tabular}

Instructions for use 


\title{
Cluster-shell competition in light nuclei
}

\author{
N. Itagaki* \\ Department of Physics, University of Tokyo, Hongo Tokyo 113-0033, Japan \\ S. Aoyama \\ Integrated Information Processing Center, Niigata University, 950-2181 Niigata, Japan \\ S. Okabe \\ Information Initiative Center, Hokkaido University, 060-0811 Sapporo, Japan \\ K. Ikeda \\ The Institute of Physical and Chemical Research (RIKEN), 351-0098 Wako, Japan
}

(Received 12 July 2004; published 5 November 2004)

\begin{abstract}
We demonstrate whether the cluster structure dissolves or remains when the shell-model-like model space is introduced in addition to the cluster model space in light nuclei. Although the binding energies of ${ }^{8} \mathrm{Be},{ }^{10} \mathrm{Be}$, and ${ }^{10} \mathrm{~B}$ become larger by about $1-2 \mathrm{MeV}$ by adding shell-model-like basis states to the $\alpha+\alpha+N+N+\cdots$ basis states, the $\alpha-\alpha$ structure is a dominant configuration of the ground states. However, $\alpha$-breaking wave functions strongly mix in ${ }^{12} \mathrm{C}$, and the decrease of the energy from the $3 \alpha$ configuration by about $6 \mathrm{MeV}$ is a clue to resolving a long-standing problem of the binding energies of ${ }^{12} \mathrm{C}$ and ${ }^{16} \mathrm{O}$. The improved version of antisymmetrized molecular dynamics (AMD), AMD superposition of selected snapshots (AMD triple-S), is used to show the cluster-shell competition of these nuclei.
\end{abstract}

DOI: 10.1103/PhysRevC.70.054307

PACS number(s): 21.30.Fe, 21.60.Cs, 27.20. $+\mathrm{n}, 27.30 .+\mathrm{t}$

\section{INTRODUCTION}

Nuclei are quantum many-body systems consisting of protons and neutrons, and these nucleons have been known to form some self-consistent mean field and to perform independent-particle motions. The nuclear shell model, which is one of the most standard models for nuclear structure, is based on this picture, and strong spin-orbit interaction in the mean field has been known to be a key mechanism to fully explain the observed magic numbers $[1,2]$.

On the other hand, the $\alpha$ particle, which corresponds to the doubly closed shell of the lowest $s$ shell in the shell model, is strongly bound, and since relative $\alpha$ - $\alpha$ interaction is weak, strongly interacting four nucleons (the $\alpha$ particle) become a subunit of the nuclear structure in some light nuclei, contrary to the mean-field picture. This molecular viewpoint has been introduced [3] even before the shell model, and such a "cluster" feature of light nuclei has been extensively studied for more than four decades [4,5]. In the socalled Ikeda diagram [6], the threshold rule has been proposed as a guiding principle to explain the appearance of the cluster structure in stable $4 N$ nuclei: a cluster structure appears around the corresponding threshold energy. Recently, theoretical and experimental investigations have proceeded to systems beyond $4 N$ nuclei, and a cluster structure with valence neutrons has become one of the main subjects concerning the structure of unstable nuclei $[7,8]$.

If an $\alpha$ cluster is expressed as the lowest $\left(s_{1 / 2}\right)^{4}$ configuration, it is a spin-zero system, and noncentral interactions do

\footnotetext{
*Electronic address: itagaki@ phys.s.u-tokyo.ac.jp
}

not contribute to its energy. However, the dissolution of the $\alpha$ cluster should be taken into account in systems where the spin-orbit interaction, which is a characteristic interaction in the mean-field picture, strongly acts. Therefore it is intriguing to study the cluster-shell competition for a unified understanding of nuclear structure.

The antisymmetrized molecular dynamics (AMD) has been proposed to demonstrate this effect and is extensively applied to calculating the properties of light stable and neutron-rich nuclei $[9,10]$. In AMD, each single particle is described as one local Gaussian $\left(G_{i}\right)$ characterized by Gaussian-center parameters $\left\{z_{i}\right\}$. The shape of the nucleus and the configuration of nucleons are determined by solving the cooling equation for these parameters. The appearance of the cluster structure and the disappearance have been discussed as a function of the neutron number, for example in the $\mathrm{Be}, \mathrm{B}$, and $\mathrm{C}$ isotopes.

However, the single AMD wave function is not sufficient to describe the quantum mechanical mixing of the shell-like configuration and the clusterlike configuration, and it is also desired to describe the "wave" nature of the nucleons well. Therefore we have proposed a new AMD approach, namely, AMD-superposition of selected snapshots (AMD triple-S) [11]. In this approach, the superposed AMD wave functions are randomly generated, and we select important ones from among them. Here, we use the idea of the stochastic variational method (SVM) [12].

In this paper, we demonstrate whether the cluster structure is dissolved or remains when the shell-model-like model space is introduced, in addition to the cluster model space, in light nuclei. For ${ }^{8} \mathrm{Be},{ }^{10} \mathrm{Be},{ }^{10} \mathrm{~B}$, and ${ }^{12} \mathrm{C}$, the persistence of the $\alpha$ - $\alpha$ structure in the ground state is studied, and for ${ }^{12} \mathrm{C}$, the persistence of the $3 \alpha$ cluster is also discussed. This paper 
is organized as follows: in Sec. II formulation is summarized, and in Sec. III, numerical results for light nuclei, cluster-shell completion is presented. The conclusion is given in Sec. IV.

\section{FRAMEWORK}

The framework of AMD triple-S is shown in Ref. [11] in detail, and here, only the important part is recaptured. The total wave function is fully antisymmetrized and is given by a superposition of the basis states (Slater determinants $\left\{\Psi_{k}\right\}$ ) with coefficients $\left\{c_{k}\right\}$ :

$$
\begin{gathered}
\Phi=\sum_{k} c_{k} P_{M K}^{J} \Psi_{k}, \\
\Psi_{k}=\mathcal{A}\left[\left(\psi_{1} \chi_{1}\right)\left(\psi_{2} \chi_{2}\right) \cdots\right]_{k} .
\end{gathered}
$$

Projection onto a good angular momentum is performed by the projection operator $P_{M K}^{J}$, and the coefficients $\left\{c_{k}\right\}$ are determined by diagonalizing the Hamiltonian matrix after this projection. Each Slater determinant $\left(\Psi_{k}\right)$ consists of $A$ single-particle states and each nucleon state $\left(\psi_{i} \chi_{i} i=1 \sim A\right)$ has a Gaussian form

$$
\psi_{i}=\left(\frac{2 v}{\pi}\right)^{3 / 4} \exp \left[-v\left(\vec{r}-\vec{z}_{i} / \sqrt{v}\right)^{2}+\vec{z}_{i}^{2} / 2\right]
$$

where $\left\{\vec{z}_{i}\right\}$ are complex parameters and $\left\{\chi_{i}\right\}$ represent the spin-isospin eigenfunctions. The oscillator parameter $(b$ $=1 / \sqrt{2 v}$ ) is set equal to $1.46 \mathrm{fm}$, which is common for all nucleons so as to get the center-of-mass kinetic energy exactly removed. The real and imaginary parts of $\vec{z}_{i}$ represent the expectation values of the position and momentum of the single particle. When we assume the presence of an $\alpha$ cluster(s), the $\vec{z}_{i}$ values are set the same for four nucleons (proton spin-up, proton spin-down, neutron spin-up, and neutron spin-down).

For each Slater determinant $\left(\Psi_{k}\right)$, the values of $\left\{\vec{z}_{i}\right\}$ are randomly generated, but we optimize the imaginary parts of these parameters to correctly take into account the spin-orbit interaction before the angular momentum projection by using the frictional cooling method in AMD. During this cooling process, the parity of the Slater determinant is projected.

After obtaining $\left\{\Psi_{k}\right\}$, the selection of the basis states is performed in $J^{\pi}$-projected space. In the case of $0^{+}$, when inclusion of a trial basis state $\Psi_{k}$ decreases the sum of the energies of the ground, second, and the third $0^{+}$states by more than $0.05 \mathrm{MeV}$, the basis states are adopted.

The Hamiltonian operator $(\hat{H})$ has the following form:

$$
\hat{H}=\sum_{i=1}^{A} \hat{t}_{i}-\hat{T}_{\mathrm{c} . \mathrm{m} .}+\sum_{i>j}^{A} \hat{v}_{i j}
$$

where a two-body interaction $\left(\hat{v}_{i j}\right)$ includes the central part, the spin-orbit part, and the Coulomb part. For the central part, we use the following Volkov no. 2 effective $N-N$ potential [13]:

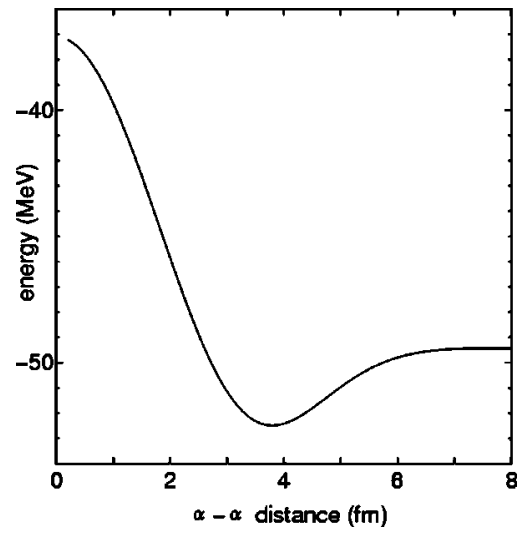

FIG. 1 . The energy curve of the $\alpha$ - $\alpha$ system $\left(0^{+}\right)$with respect to the distance between the centers of the two $\alpha$ clusters. The energy does not coincide with twice the energy of the $\alpha$ cluster $(2 \times$ $-27.57 \mathrm{MeV}$ ) even at a large $\alpha$ - $\alpha$ distance, since the relative distance is fixed. The corresponding kinetic energy of the zero-point oscillation is $\hbar \omega / 4=4.86 \mathrm{MeV}$.

$$
\begin{aligned}
V(r)= & \left(W-M P^{\sigma} P^{\tau}+B P^{\sigma}-H P^{\tau}\right) \\
& \times\left[V_{1} \exp \left(-r^{2} / c_{1}^{2}\right)+V_{2} \exp \left(-r^{2} / c_{2}^{2}\right)\right],
\end{aligned}
$$

where $W=1-M, M=0.60$, and $B=H=0.125$. For the spinorbit term, we introduce the G3RS potential [14] as

$$
V_{l s}=V_{0}\left\{e^{-d_{1} r^{2}}-e^{-d_{2} r^{2}}\right\} P\left({ }^{3} O\right) \vec{L} \cdot \vec{S},
$$

where $d_{1}=5.0 \mathrm{fm}^{-2}, d_{2}=2.778 \mathrm{fm}^{-2}, V_{0}=2000 \mathrm{MeV}$, and $\mathrm{P}\left({ }^{3} \mathrm{O}\right)$ is a projection operator onto a triplet odd state. The operator $\vec{L}$ stands for the relative angular momentum and $\vec{S}$ is the spin $\left(\vec{S}_{1}+\vec{S}_{2}\right)$. All of the parameters of this interaction were determined from the $\alpha+n$ and $\alpha+\alpha$ scattering phase shifts and the binding energy of the deuteron [15]. The original Volkov no. 2 potential gives a bound state for the $n-n$ system, but that is eliminated by introducing the $B$ and $H$ parameters.

\section{RESULTS}

\section{A. $\alpha$ - $\alpha$ energy curve}

One of the most basic cluster structures in light nuclei is the $\alpha$-cluster structure, and the energy curve of the $\alpha+\alpha$ system $\left(0^{+}\right)$calculated by using the present effective interaction (Volkov no. 2, $M=0.6$ ) is shown in Fig. 1.

The energy becomes minimum around the $\alpha$ - $\alpha$ distance of $3.5 \mathrm{fm}$, and it increases rapidly at smaller distances. At the limit of zero distance, where the wave function corresponds to the $(s)^{4}(p)^{4}$ configuration of the shell model, the energy is higher by $15 \mathrm{MeV}$ compared with the lowest energy. Therefore, in general, it is very difficult to shrink the $\alpha-\alpha$ distance so as to eliminate the $\alpha$ - $\alpha$ cluster structure, even if valence nucleons are added to the $\alpha+\alpha$ system and $\alpha$ clusters are more attracted to each other. For example, we have analyzed ${ }^{10} \mathrm{Be}$ by using the same interaction by introducing the $\alpha+\alpha$ $+n+n$ model [16], and although the increase of the binding energy from ${ }^{8} \mathrm{Be}$ is about $8 \mathrm{MeV}$, the optimal $\alpha$ - $\alpha$ distance 


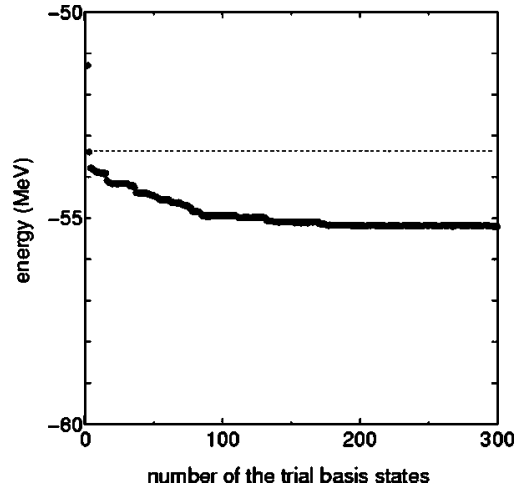

FIG. 2. The energy convergence of ${ }^{8} \mathrm{Be}\left(0^{+}\right)$with respect to the number of trial AMD basis states. The first three basis states have the $\alpha+\alpha$ structure with the relative distances of 2,3 , and $4 \mathrm{fm}$. The energy calculated using these three is shown as the dotted line, and the basis states of the $\alpha+p+p+n+n$ model space are included in the calculations represented by the lower-lying dots.

remains at around $3 \mathrm{fm}$ and the cluster structure survives. Therefore if the $\alpha$ cluster disappears, it can be related more to the dissolution of an $\alpha$ cluster than to the shrinking effect due to the attractive interaction caused by valence nucleons. The spin-orbit interaction is one of the candidates for the origin of the dissolution of $\alpha$ clusters, and we concentrate on it in the following subsections.

\section{B. ${ }^{8} \mathbf{B e}$}

The ${ }^{8} \mathrm{Be}$ nucleus has been known as a typical example of the $\alpha$-cluster structure. This molecular structure has been also confirmed by recent quantum Monte Carlo calculation with a realistic nucleon-nucleon interaction [17]. We also check that the $\alpha$-cluster structure dominates the structure of the ground state of ${ }^{8} \mathrm{Be}$. In our model, the wave functions with the $\alpha$ - $\alpha$ distances of 2, 3, and $4 \mathrm{fm}$ are prepared, and, in addition to these cluster basis states, the shell model component is introduced.

The energy convergence of ${ }^{8} \mathrm{Be}\left(0^{+}\right)$with respect to the number of the trial AMD basis states is shown in Fig. 2. The first three basis states have the $\alpha+\alpha$ structure with the relative distances of 2,3 , and $4 \mathrm{fm}$, which gives $-53.4 \mathrm{MeV}$ shown as the dotted line, and we add wave functions of the $\alpha+p+p+n+n$ model space corresponding to the shellmodel-like basis states. Here, the values of Gaussian-center parameters of four valence nucleons are randomly generated, and only their imaginary parts are optimized by solving the cooling equation. By adding these shell-model-like states, the energy decreases by about $1.8 \mathrm{MeV}$ from that of the $\alpha$ - $\alpha$ model space (relative distances of 2, 3, and $4 \mathrm{fm}$ ). This decrease of the energy should be said to be rather small, because it includes the effects of the improvement of an $\alpha$-cluster wave function with respect to $\left(s_{1 / 2}\right)^{4}$ (the energy decrease is more than $1 \mathrm{MeV}$ ) and their coupling with $\alpha$ - $\alpha$ states with very small distance. Therefore the $\alpha$ - $\alpha$ cluster structure is still a dominant component of the ground state. The squared overlap between the final result and the lowest state obtained from the first three basis states is 0.94 . This

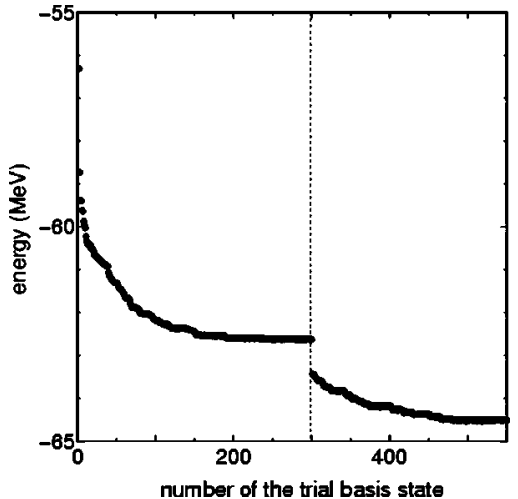

FIG. 3. The energy convergence of ${ }^{10} \mathrm{Be}\left(0^{+}\right)$with respect to the number of trial AMD wave functions. The basis states from 1 to 300 are those of the $\alpha+\alpha+n+n$ model space with the relative $\alpha$ - $\alpha$ distance of 2, 3, and $4 \mathrm{fm}$, and after 300, shell-model-like wave functions of the $\alpha+2 p+4 n$ model space are added.

shows the cluster structure persists in the ground state of ${ }^{8} \mathrm{Be}$, although the cluster-breaking (shell-model-like) component mixes by several per cent. Hereafter, we refer to the decrease of the energy when the breaking up of one of the $\alpha$ clusters is taken into account as $\Delta$.

\section{C. ${ }^{10}$ Be and ${ }^{10} \mathrm{~B}$}

We examine the persistence of the cluster structure when the $\alpha$ clusters are more strongly bound to each other due to additional valence nucleons. Similarly to the ${ }^{8} \mathrm{Be}$ case, the energy convergence of ${ }^{10} \mathrm{Be}\left(0^{+}\right)$with respect to the number of trial AMD wave functions is shown in Fig. 3. The basis states from 1 to 300 are those of the $\alpha+\alpha+n+n$ model space with the relative $\alpha$ - $\alpha$ distance of 2,3 , and $4 \mathrm{fm}$, and after 300 , the shell-model-like wave functions of the $\alpha+2 p+4 n$ model space are added. The energy converges at $-64.5 \mathrm{MeV}$ (the experimental value is $-65.0 \mathrm{MeV}$ ), and the decrease with respect to the $\alpha+\alpha+n+n$ model space by adding the $\alpha+2 p+4 n$ model space $(\Delta)$ is $1.9 \mathrm{MeV}$. This decrease is almost the same as in ${ }^{8} \mathrm{Be}$, and it is concluded that the breaking of the clusters is more or less at the same level.

By changing one valence neutron to a proton, the energy convergence of ${ }^{10} \mathrm{~B}\left(3^{+}\right)$with respect to the number of trial AMD basis states is shown in Fig. 4. Similarly, $\Delta$ is found to be about $1.9 \mathrm{MeV}$.

\section{D. ${ }^{12} \mathrm{C}$}

There have been a lot of successful calculation for ${ }^{12} \mathrm{C}$ assuming the $3 \alpha$ configuration $[18,19]$, and the second $0^{+}$ state just above the $3 \alpha$ threshold has been known to be well described by the cluster models. However, the breaking effect of an $\alpha$ cluster has been also known to be important, especially for the ground state [20-22], to explain the observed electromagnetic properties. Here, we investigate this cluster-shell mixing in detail, and the Majorana parameter of the Volkov no. 2 interaction is changed to 0.62 to reproduce the binding energy. 


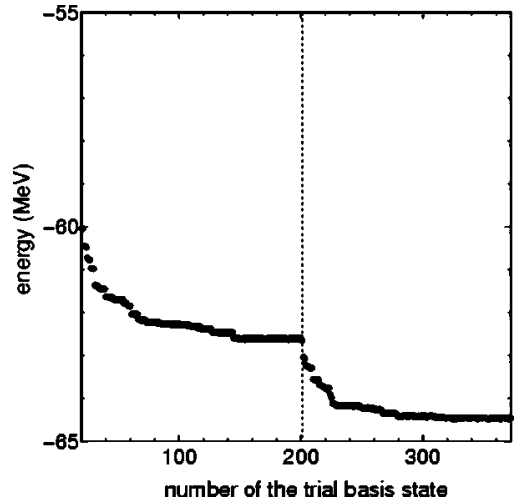

FIG. 4. The energy convergence of ${ }^{10} \mathrm{~B}\left(3^{+}\right)$with respect to the number of trial AMD wave functions. The basis states from 1 to 200 are those of the $\alpha+\alpha+p+n$ model space with the relative $\alpha$ - $\alpha$ distance of 2, 3, and $4 \mathrm{fm}$, and after 200, shell-model-like wave functions of the $\alpha+3 p+3 n$ model space are added.

At first, we show the energy curve of ${ }^{12} \mathrm{C}$ with respect to the expectation value of the principal quantum number $(\langle\vec{a} \cdot \vec{a}\rangle)$, by using a single AMD wave function. Here, both the real and imaginary parts of the Gaussian centers are optimized under the constraint of the $\left\langle\vec{a}^{\dagger} \cdot \vec{a}\right\rangle$ values. In Fig. 5, the solid line represents the result with the spin-orbit term, and the dotted line shows the result without it. At the lowest value of 8 (eight nucleons are in the $p$ shell), the $j j$-coupling picture of the shell model is important, and the energy difference between the two lines is very large (about $10 \mathrm{MeV}$ ). The dissolution of the $\alpha$ cluster(s) occurs at the lowest principal quantum number (shell-model limit). However, at larger values $(\sim 12)$, the difference between the two lines becomes much smaller, because of the formation of three $\alpha$ clusters. For the interpretation of the solid curve, the presence of these two structures (shell structure at $\left\langle\vec{a}^{\dagger} \cdot \vec{a}\right\rangle \sim 8$ and $3 \alpha$-cluster structure at larger values) has to be taken into account.

Next, AMD triple-S is applied, and the energy convergence of ${ }^{12} \mathrm{C}$ is shown in Fig. 6 . The basis states from 1 to 100 have various configurations of $3 \alpha$, and those from 101 to

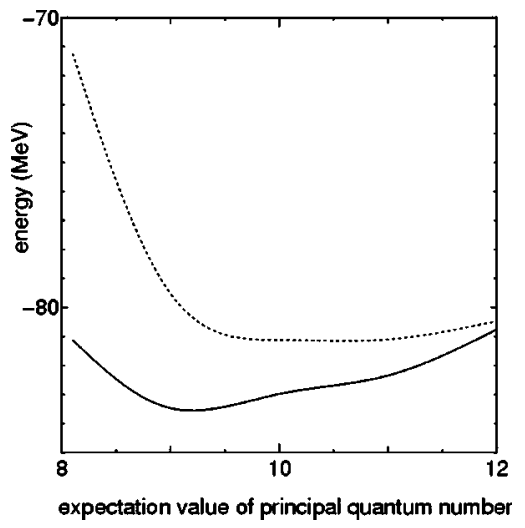

FIG. 5. The energy curve of ${ }^{12} \mathrm{C}$ with respect to the expectation value of the principal quantum number $\left(\vec{a}^{\dagger} \cdot \vec{a}\right)$. The solid line and dotted line represent the results with the spin-orbit interaction and without it, respectively.

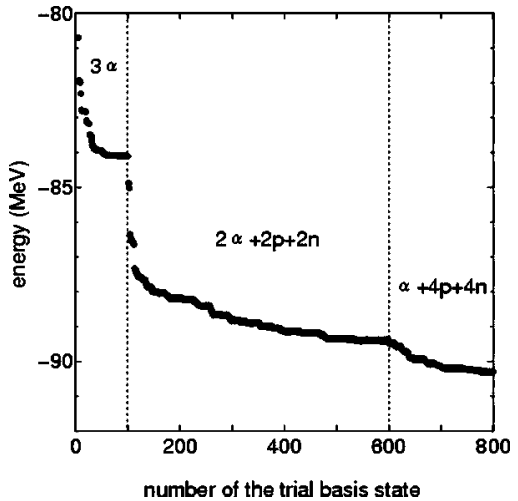

FIG. 6. The energy convergence of ${ }^{12} \mathrm{C}\left(0^{+}\right)$with respect to the number of trial AMD basis states. The basis states from 1 to 100 are those of the $3 \alpha$ model space, and those from 101 to 600 are $\alpha+\alpha$ $+2 p+2 n$ model space with relative $\alpha-\alpha$ distances of 2,3 , and $4 \mathrm{fm}$. After 601, the wave functions with the shell-model-like $\alpha+4 p$ $+4 n$ model space are added.

600 have $\alpha+\alpha+2 p+2 n$ model space (relative $\alpha$ - $\alpha$ distances of 2,3 , and $4 \mathrm{fm}$ ). Here, the decrease of energy with respect to the $3 \alpha$ model is $5.2 \mathrm{MeV}$ due to the strong spin-orbit interaction. Furthermore, the shell-model-like wave functions of the $\alpha+4 p+4 n$ model space are added (from 601 to 800), where two of $\alpha$ clusters are broken. However, the decrease of the energy by adding these basis states is only about $1 \mathrm{MeV}$, and it is seen that the contribution of the spinorbit interaction is almost taken into account with one of $\alpha$ clusters broken. The squared overlaps between the ground state of the final solution and the lowest, the second, and the third $0^{+}$states of the $3 \alpha$-cluster configuration (1-100 basis states) are $0.56,0.06,0.01$, respectively. Therefore, although the ground states has the $3 \alpha$ component of about $60 \%$, a large amount of $\alpha$ breaking component is mixed in.

On the other hand, the calculated second $0^{+}$state (observed at $E_{x}=7.65 \mathrm{MeV}$ ) mainly has the $3 \alpha$ component. The sum of the squared overlaps between the second $0^{+}$state and the lowest three $0^{+}$states of $3 \alpha$-cluster configuration exceeds 0.8 . This state is a typical cluster state, and the shell-model study by Cohen and Kurath [23] gives too high excitation energy (13-14 MeV) for the state, suggesting that it is out of the model space. In stellar nucleosynthesis, the second $0^{+}$ state just above the $3 \alpha$ threshold plays a crucial role in forming ${ }^{12} \mathrm{C}$. It is a triple- $\alpha$ resonance state and decays first to the $2^{+}$state and next to the $0^{+}$ground state by emitting $\gamma$ rays. The $B\left(E 2,0_{2}^{+} \rightarrow 2_{1}^{+}\right)$value strongly affects the abundance of ${ }^{12} \mathrm{C}$, however, it has been underestimated by a factor of 2 in the traditional $3 \alpha$ model based on the resonating group method (RGM) [18]. As shown in Table I, in the present case the mixing of $3 \alpha$ and the $\alpha$ breaking components affects the $B(E 2)$ value and it becomes consistent with the experimental value, in the same way as our previous analysis based on the molecular-orbital model [22].

Furthermore, the present result gives a clue to a long standing problem of the binding energies of ${ }^{12} \mathrm{C}$ and ${ }^{16} \mathrm{O}$. It has been known that in microscopic cluster models, when we use the effective interaction which reproduces the binding energy of the $3 \alpha$ system, the $4 \alpha$-system becomes over-bound 
TABLE I. The electromagnetic transition probability from the $2^{+}$state to the ground state and one from the second $0^{+}$state to the $2^{+}$state (left column). They are compared with the experimental values (middle column) and the $3 \alpha$-RGM calculation [18] (right column). All units are $e^{2} \mathrm{fm}^{4}$.

\begin{tabular}{cccc}
\hline \hline & Present & Expt. & $3 \alpha$ RGM \\
\hline $2_{1}^{+} \rightarrow 0_{1}^{+}$ & 7.1 & $7.8 \pm 0.4$ & 9.3 \\
$0_{2}^{+} \rightarrow 2_{1}^{+}$ & 14.1 & $13 \pm 4$ & 5.6 \\
\hline \hline
\end{tabular}

by about $20 \mathrm{MeV}$. On the contrary, if the binding energy of $4 \alpha$ is reproduced, the $3 \alpha$ system becomes underbound by about $10 \mathrm{MeV}$. We had previously discussed that the experimental binding energy difference between ${ }^{12} \mathrm{C}$ and ${ }^{16} \mathrm{O}$ cannot be fully reproduced, even if we utilize a finite-range and density-dependent interaction [24], when the model space is restricted to $\alpha$ clusters. Now it is shown that by incorporating the $\alpha$-breaking component, the binding energy of ${ }^{12} \mathrm{C}$ becomes deeper by several $\mathrm{MeV}$ due to the spin-orbit interaction. Since this effect is considered to be less important for ${ }^{16} \mathrm{O}$, which has a doubly closed configuration of the $p$ shell, the spin-orbit interaction would be one of the important keys to resolve this long-standing problem. However, we have a preliminary result that only half of the binding-energy problem can be solved by incorporating the spin-orbit interaction, and consideration of remaining effects, such as direct treatment of the tensor interaction, whose effect is renormalized in the central and spin-orbit parts of the interaction, would play a role in fully solving the problem.

The coupling effects between the cluster states and shellmodel states are summarized as a "nuclear chart" of the cluster-shell competition in Fig. 7. Here, $\Delta$ represents the increase of the binding energy when the breaking of one of the $\alpha$ clusters is taken into account. The nuclei ${ }^{8} \mathrm{Be},{ }^{10} \mathrm{Be}$, and ${ }^{10} \mathrm{~B}$ have the $\Delta$ values of about $2 \mathrm{MeV}$, and the $\alpha+\alpha$ structure is the dominant configuration of the ground states. However, the $\Delta$ value is very large in ${ }^{12} \mathrm{C}(5.2 \mathrm{MeV})$. For ${ }^{12} \mathrm{C}, \Delta(2)$ is also shown, which is the increase of the binding energy when the breaking of two of the $\alpha$ clusters is taken into account $(6.2 \mathrm{MeV})$.

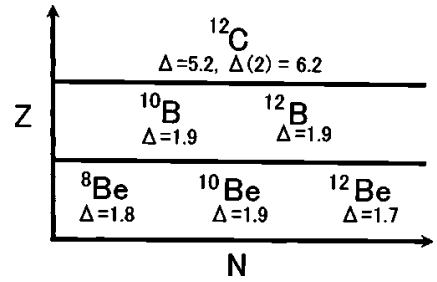

FIG. 7. The nuclear chart of the cluster-shell competition. $\Delta$ represents the increase of the binding energy when the breaking of one of the $\alpha$ clusters is taken into account. For ${ }^{12} \mathrm{C}, \Delta(2)$ is also shown, which is the increase of the binding energy when the breaking of two of the $\alpha$ clusters is taken into account.

\section{CONCLUSION}

We have discussed the cluster-shell competition in light nuclei, by using the AMD triple-S model. Now it becoms possible to prepare cluster states and $\alpha$ breaking (shellmodel-like) states on the same footing and to calculate the mixing of these states by using a common effective interaction.

It has been shown that the binding energies of ${ }^{8} \mathrm{Be},{ }^{10} \mathrm{Be}$, and ${ }^{10} \mathrm{~B}$ become larger by adding shell-model-like basis states, but the amount is only about $2 \mathrm{MeV}$, and the $\alpha+\alpha$ structure is the dominant configuration in the ground state. However, the decrease of the energy from the $3 \alpha$-cluster state due to the spin-orbit interaction is very large in ${ }^{12} \mathrm{C}$ (about $6 \mathrm{MeV}$ ). This strongly suggests the dissolution of $\alpha$ cluster(s) in the ground state, and the mixing of cluster and shell components makes the $B(E 2)$ values consistent with the experimental values, which are important in nuclear astrophysics. The present result also gives an insight into the resolution of the long standing problem of the binding energies of ${ }^{12} \mathrm{C}$ and ${ }^{16} \mathrm{O}$. However, consideration of remaining effects, such as direct treatment of the tensor interaction, would be important to fully solve the problem.

\section{ACKNOWLEDGMENTS}

This work was supported in part by a Grant-in-Aid for Scientific Research (Grant Nos. 14740142 and 15740141) from the Ministry of Education, Science and Culture. Also, this work was partially performed in the Research Project for Study of Unstable Nuclei from Nuclear Cluster Aspects sponsored by Institute of Physical and Chemical Research (RIKEN).
[1] O. Haxel, J.H.D. Jensen, and H.E. Suess, Phys. Rev. 75, 1766 (1949).

[2] M.G. Mayer, Phys. Rev. 75, 1969 (1949).

[3] J.A. Wheeler, Phys. Rev. 52, 1083 (1937).

[4] D.M. Brink, Proceedings of the International School of Physics "Enrico Fermi," Course XXXVI, edited by C. Bloch (Academic, New York, 1966), p. 247.

[5] Y. Fujiwara, H. Horiuchi, K. Ikeda, M. Kamimura, K. Katō, Y. Suzuki, and E. Uegaki, Suppl. Prog. Theor. Phys. 68, 60 (1980)

[6] K. Ikeda, N. Takigawa, and H. Horiuchi, Suppl. Prog. Theor.
Phys. , Extra number, 464 (1968).

[7] W. von Oertzen, Z. Phys. A 354, 37 (1996); 357, 355 (1997).

[8] M. Freer et al., Phys. Rev. Lett. 82, 1383 (1999).

[9] Y. Kanada-En'yo and H. Horiuchi, Prog. Theor. Phys. 93, 115 (1995).

[10] M. Kimura, Y. Sugawa, and H. Horiuchi, Prog. Theor. Phys. 106, 1153 (2001).

[11] N. Itagaki, A. Kobayakawa, and S. Aoyama, Phys. Rev. C 68, 054302 (2003).

[12] K. Varga, Y. Suzuki, and Y. Ohbayasi, Phys. Rev. C 50, 189 (1994). 
[13] A.B. Volkov, Nucl. Phys. 74, 33 (1965).

[14] N. Yamaguchi, T. Kasahara, S. Nagata, and Y. Akaishi, Prog. Theor. Phys. 62, 1018 (1979).

[15] S. Okabe and Y. Abe, Prog. Theor. Phys. 61, 1049 (1979).

[16] N. Itagaki and S. Okabe, Phys. Rev. C 61, 044306 (2000).

[17] R. B. Wiringa, Steven C. Pieper, J. Carlson, and V. R. Pandharipande, Phys. Rev. C 62, 014001 (2000).

[18] M. Kamimura, Nucl. Phys. A351, 456 (1981).

[19] Y. Funaki, A. Tohsaki, H. Horiuchi, P. Schuck, and G. Röpke,
Phys. Rev. C 67, 051306 (2003).

[20] N. Takigawa and A. Arima, Nucl. Phys. A168, 593 (1971).

[21] Y. Kanada-En'yo, Phys. Rev. Lett. 81, 5291 (1998).

[22] N. Itagaki, K. Hagino, T. Otsuka, S. Okabe, and K. Ikeda, Nucl. Phys. A719, 205c (2003).

[23] S. Cohen and D. Kurath, Nucl. Phys. A73, 1 (1965).

[24] N. Itagaki, A. Ohnishi, and K. Katō, Prog. Theor. Phys. 94, 1019 (1995). 